\title{
Vaccinations for Neuroinfectious Disease: A Global Health Priority
}

\author{
Emily C. Leibovitch ${ }^{1,2} \cdot$ Steven Jacobson ${ }^{1}$
}

Published online: 30 June 2016

(C) The American Society for Experimental NeuroTherapeutics, Inc. (outside the U.S.) 2016

\begin{abstract}
Vaccines for neuroinfectious diseases are becoming an ever-increasing global health priority, as neurologic manifestations and sequelae from existing and emerging central nervous system infections account for significant worldwide morbidity and mortality. The prevention of neurotropic infections can be achieved through globally coordinated vaccination campaigns, which have successfully eradicated nonzoonotic agents such as the variola viruses and, hopefully soon, poliovirus. This review discusses vaccines that are currently available or under development for zoonotic flaviviruses and alphaviruses, including Japanese and tick-borne encephalitis, yellow fever, West Nile, dengue, Zika, encephalitic equine viruses, and chikungunya. Also discussed are nonzoonotic agents, including measles and human herpesviruses, as well as select bacterial, fungal, and protozoal pathogens. While therapeutic vaccines will be required to treat a multitude of ongoing infections of the nervous system, the ideal vaccination strategy is preexposure vaccination, with the ultimate goals of minimizing disease associated with zoonotic viruses and the total eradication of nonzoonotic agents.
\end{abstract}

Electronic supplementary material The online version of this article (doi:10.1007/s13311-016-0453-3) contains supplementary material, which is available to authorized users.

Steven Jacobson

jacobsons@ninds.nih.gov

1 Viral Immunology Section, National Institute of Neurological Disorders and Stroke, National Institutes of Health, Bethesda, MD 20892, USA

2 Institute for Biomedical Sciences, The George Washington University School of Medicine, Washington, DC 20037, USA
Keywords Vaccines $\cdot$ Neuroinfectious $\cdot$ Neuroinvasive Meningitis $\cdot$ Encephalitis $\cdot$ CNS infections

\section{Introduction}

The World Health Organization (WHO) estimates that vaccination is only second to clean water as a major preventative measure of human infectious disease. Annually, vaccines prevent around 6 million deaths worldwide [1], and from a public health standpoint are extraordinarily cost-effective. As neurologic manifestations and sequelae from existing and emerging central nervous system (CNS) infections account for significant worldwide morbidity and mortality, the development and adequate distribution of vaccines for neuroinfectious diseases should be prioritized [2]. CNS infections can result in symptoms ranging from fever and increased intracranial pressure to disorientation and altered mental status, which, if not fatal, are associated with permanent residual deficits. Moreover, virtually every age group is susceptible to CNS infectious disease, from neonates to older adults [3].

The prevention of neurotropic infections can be achieved through globally coordinated vaccination campaigns. This review aims to highlight several major classes of neuroinfectious agents and the status of the associated vaccination efforts (for an excellent overview of global CNS infections, see [4]). Many of the most globally significant neuroinfectious agents are zoonotic arboviruses, which are the primary focus of this review; however, nonzoonotic viruses and select nonviral agents are also discussed.

\section{Vaccines Available Against Zoonotic Flaviviruses}

Zoonotic infections are those transmitted from vertebrate animals to humans directly or indirectly, for example by a blood- 
feeding arthropod vector intermediate [5]. Arthropod-borne viruses are predominantly RNA viruses that replicate in arthropods prior to transmission [6]. As these agents have nonhuman reservoirs, an achievable goal is effective control of the spread of infection via prophylactic, or pre-exposure vaccination. The mature virion of all flaviviruses consists of an icosahedral particle with three structural proteins: capsid, membrane, and envelope [7]. The primary target of neutralizing antibodies induced by flavivirus vaccines is the envelope protein, although antibodies against the membrane protein and a nonstructural protein, NS1, have also been shown to be protective [8].

\section{Japanese Encephalitis Virus}

Japanese encephalitis (JE) is the leading vaccine-preventable cause of encephalitis in Asia, with an estimated 68,000 annual cases worldwide [9]. The natural reservoirs of JE virus (JEV) are pigs and wading birds, and the virus is transmitted to humans by Culex mosquitos, primarily in rural agricultural settings [9]. The majority of infections are asymptomatic; < $1 \%$ of infected individuals develop clinical disease, and the most commonly recognized manifestation is acute encephalitis. Other presentations include aseptic meningitis, seizures, or acute flaccid paralysis. The fatality rate is $20-30 \%$, and 30 $50 \%$ survivors are left with neurologic sequelae [10].

Several classes of JEV vaccines are available, including inactivated, live attenuated and live recombinant versions [11]. All vaccines are based on strains of the GIII JEV genotype, and current data suggest that vaccination can induce crossprotective immunity against all major JEV genotypes [9]. The full duration of protection is unknown, however, studies suggest that antibodies are maintained for approximately two years. Between 1973 and 2013, 68 cases of JE were published or reported to the Centers for Disease Control, but only eight were reported between 1993 and 2013, during which a vaccine became available [10]. Moreover, endemic countries that have implemented routine childhood vaccinations have recorded remarkable declines in disease incidence [9].

\section{Tick-Borne Encephalitis Virus}

Tick-borne encephalitis (TBE) virus is the most common arthropod-transmitted viral infection of humans in Europe and central and eastern Asia [12]. Of the three known subtypes, the Siberian and Far-Eastern subtypes are associated with comparatively more severe disease than the European subtype [13]. Clinical manifestations range from fever, headache and nausea to encephalitis with or without altered consciousness [14]. One study of the Polish health registry reported that TBE presents as meningitis in about $50 \%$ patients, meningoencephalitis in about $40 \%$ and meningoencephalomyelitis in about $10 \%$ [13]. Neurologic sequelae have been reported in up to $50 \%$ affected individuals, particularly neurocognitive and motor symptoms. The disease is fatal in approximately $1-4 \%$ cases; poorer outcomes are usually observed among elderly patients and those with chronic diseases or additional infections.

As transmission occurs through bite of an infected tick, there is no direct person-to-person transmission of TBEV [11]. Forest workers and others with a high work-related risk of infection are often vaccinated [13]. Austria boasts the world's highest general population vaccination rate $(82 \%)$ and produces one of the two licensed vaccines available in Europe. Both of these vaccines contain purified TBE virus grown in chick embryo cells, inactivated by formalin, with an aluminum hydroxide adjuvant [12] and appear to protect against all TBEV subtypes circulating in Europe and Asia [15].

\section{Yellow Fever Virus}

Yellow fever (YF) occurs in sub-Saharan Africa and Central and South America. Most infections are asymptomatic, though a subset can lead to jaundice, vomiting, and hemorrhagic manifestations. Yellow fever is also associated with encephalitis and myelitis [16]. Approximately 200,000 cases of YF occur annually, leading to 60,000 deaths worldwide [7, 17]. Transmission occurs through Aedes aegypti mosquitos. In rural areas, monkeys are the main reservoir, and mosquitos spread the disease between monkeys and humans in a cycle referred to as jungle yellow fever (sylvatic). In urban areas, mosquitos can transmit the virus between humans in a cycle known as urban yellow fever.

The currently available vaccine, YF-17D, has been administered to more than 600 million people worldwide and is live attenuated, developed by successive passages of the Asibi viral strain in mouse and chicken embryos [7]. A single dose should confer lifelong protective immunity [18], which is reflected by the vaccine's strong immune signature; notably, the efficient activation of antigen presenting cells, high neutralizing antibody titers, the induction of polyfunctional $\mathrm{T}$ cells, and long-term memory T and B cells [7]. A robust type I interferon response can be transiently observed in the blood transcriptomes of vaccinated individuals [19]. Owing to its strong elicitation of immune responses, the yellow fever vaccine has become a vector platform for vaccine development of heterologous viruses such as JEV and dengue.

Yellow fever outbreaks still occur despite the available vaccine, as evidenced by the ongoing outbreak in Angola, the largest in almost 30 years. The world's annual YF vaccine production is around 40 million doses, which, while sufficient for small outbreaks in Africa, would be entirely insufficient if the disease became established in Asia. However, even in Africa, vaccination rates must be improved, as some countries have less than $50 \%$ coverage. In the opinion of a WHO official, the availability of a YF vaccine may have ironically contributed to complacency in vector control and other preventative measures [17]. 


\section{Vaccines Under Development for Zoonotic Flaviviruses}

\section{West Nile Virus}

Clinical manifestations of West Nile virus (WNV) can include fever, headache, seizures, and generalized paresis. WNV first emerged in the USA in 1999, and over the next ten years there were 12,000 reported cases of West Nile neuroinvasive disease, with a mortality rate of approximately $10 \%$ [20].

There are several live and inactivated WNV vaccines approved for equines, but none yet for humans. The JE serocomplex is comprised of nine genetically and antigenically related flaviviruses, including WNV [21]. Research has demonstrated that infection with one JE serocomplex virus can provide protection against heterologous viruses, raising the question of harnessing cross-protection for WNV from existing JEV vaccines. In a 2013 study, Petrovsky et al. [20] demonstrated that in mice, JE-ADVAX induced complete cross-protection of WNV, with a reduced viral burden and no CNS perivascular lymphocytic infiltrates. In this study, WNV protection appeared to be mediated by the induction of cross-protective memory B cells [20]. The JE-ADVAX vaccine is a Vero cell-derived, formalin-inactivated JEV antigen with an Advax adjuvant [20]. Vero cells were originally derived from African green monkey kidney cells and are a widely used platform in vaccine production [21]. Two human studies used an inactivated JE vaccine (JE-VAX) to examine the induction of cross-neutralizing WNV antibodies. While one study reported negative results, another that co-delivered the JE vaccine with the YF-17D vaccine reported effective levels of WNV cross-neutralizing antibodies, which waned faster than JE antibodies but increased again after a booster with JE-VAX [22].

\section{Dengue Virus}

Dengue infects around 390 million people annually, most commonly in South Asia, Africa, and Latin America. Similar to yellow fever, chikungunya, and Zika, the principal vector is the A. aegypti mosquito [23]. Neurologic outcomes of infection can include meningitis, meningoencephalitis, encephalitis, seizures, and Guillain-Barré syndrome [4].

Dengue can be divided into four serotypes, which share $67 \%$ homology at the nucleotide level and differ by up to $37 \%$ in the envelope protein sequences. In 2007, a distinct serotype was isolated from Malaysia; though phylogenetically distinct from all known DENV serotypes, it remains unclear whether it represents a fifth serotype or a variant. Patients who are sequentially infected by multiple DENV serotypes are more likely to manifest severe symptoms, which is thought to be attributable to the mechanism of antibody-dependent enhancement. Therefore, a safe and effective dengue vaccine must be multivalent to induce robust homotypic immunity to each of the four serotypes [7, 24].

Despite attempts at vaccine development over the last 70 years [24], only in December 2015 was the first dengue vaccine licensed in Mexico [25]. This CYD-TDV vaccine is a live-attenuated formulation, as are the two other candidates awaiting Phase 3 evaluation. CYD-TDV is tetravalent, produced by inserting DENV genes from each of the four serotypes into the YF-17D backbone. Initial results suggested that neutralizing antibodies were unequally elicited to the four serotypes; DENV-3 and DENV-4 elicited the most robust seroconversion frequencies and virema compared to DENV-1 and DENV-2 [24]. There was also limited induction of an early proinflammatory response and CD8 T-cell activation [7]. This may be because the monovalent components of the vaccine contain nonstructural proteins of the yellow fever 17D virusnot DENV - and many DENV CD8 epitopes are located in nonstructural proteins. Again, balanced infectivity of all four components is essential for the induction of homotypic antibody to each serotype [24], which remains the ultimate goal of a DENV vaccine in the context of known antibody-dependent enhancement.

\section{Zika Virus}

Zika is a flavivirus that has been recognized in Africa and Asia since the 1940s, but the virus's geographic range has greatly expanded since 2007 . The principal vectors are $A$. aegypti mosquitos [26], and there is increasing evidence that Zika may also be transmitted sexually [27]. Infection is asymptomatic in about $80 \%$ cases, and causes a mild disease in the other $20 \%$, characterized by fever, muscle aches, eye pain, and maculopapular rash [28]. The virus is also neurotropic, as evident from the surge in microcephalic births in areas affected by the 2015 South American outbreak [29]. In a Brazilian cohort of 35 infants with microcephaly, all mothers had been in Zika-affected areas during pregnancy; $71 \%$ infants had severe microcephaly, $50 \%$ had at least one neurologic abnormality, and among 27 infants with neuroimaging, all had abnormalities [30]. Moreover, Zika virus RNA has been isolated from the amniotic fluid of women with microcephalic fetuses, as well as from the brains of several deceased infants with microcephaly.

In addition to microcephaly, there are reports of other neurologic complications resulting from Zika, including GuillainBarré syndrome [6], which was reported in approximately 1 per 4000 infections in the 2013 French Polynesia outbreak $[31,32]$, and reports of meningoencephalitis and acute myelitis [33, 34]. Murine experiments in the early 1970s suggested Zika's neurotropism, as intracerebral inoculation of mice resulted in neuronal destruction, with viral replication observed in neurons and astrocytes [35]. 
There are no Zika vaccines yet in advanced development [6], though there are reports that safety trials may begin in the fall of 2016, and efficacy trials as early as 2017. A livevectored formulation may have the greatest success, given Zika's similarity to YF, JE, and dengue viruses [27]. As the rubella vaccine eliminated congenital rubella syndrome in countries where it was widely administered, there is an incentive to deploy a Zika virus vaccine quickly. The ideal vaccine should rapidly protect against infection after a single dose, and be safe to administer during pregnancy [36].

\section{Vaccines Under Development for Zoonotic Alphaviruses}

\section{Encephalitic Equine Viruses}

The encephalitic alphaviruses Eastern, Western, and Venezuelan equine encephalitis viruses (EEEV, WEEV and VEEV) are widely distributed throughout North, Central, and South America. In North America, WEEV is found from the mid-west to the west coast, while EEEV is found from the mid-west to the Gulf and Atlantic coasts. VEEV is found predominantly in Central and South America [37].

The viruses have differing fatalities among humans and horses, but all are more significantly fatal to horses. In humans, EEEV is the most virulent of the three, with an estimated mortality rate of 50-70\% (WEEV, 3-7\%) and (VEEV, $1 \%$ ) [37]. EEEV can cause symptoms of encephalitis, fever, headache, and seizures. Brain edema with necrosis and hemorrhage are seen upon gross pathological examination of fatal human cases. By contrast, though a minority of individuals infected with WEEV or VEEV experience encephalitis or encephalomyelitis, these viruses are associated with significant neurologic sequelae [37, 38]. In a 1995 VEEV outbreak in Columbia and Venezuela, of 313 hospitalized patients, over half had signs of intracranial hypertension, and neurologic complications including cerebellitis, meningoencephalitis, and encephalomyelitis [39].

The live attenuated VEEV strain TC-83 is the only licensed veterinary vaccine available to protect equines, and the only strain available for vaccinating laboratory workers and military personnel [40]. Nearly $40 \%$ of vaccinated individuals develop a disease with some symptoms of VEEV infection [38] and in equines, TC-83 vaccination can lead to viremia levels sufficient to infect mosquitos [38, 40], likely attributable to the presence of only two attenuating point mutations. In an effort to create a more safe and stable VEEV vaccine, Guerbois et al. [38] generated a recombinant candidate that does not infect mosquitos, and is stable following several passages [38].

As current vaccine strategies target only a single alphavirus species (VEEV), Phillips et al. [41] designed an alphavirus vaccine comprised of cationic lipid nucleic acid complexes and the ectodomain of the WEEV E1 protein. This immunization provided $100 \%$ protection in mice challenged with WEEV subcutaneously, intranasally, or via mosquito. Moreover, $90 \%$ of the mice survived EEEV challenge, demonstrating strong cross-protection of the WEEV immunization and the potential to develop a pan-encephalitic alphavirus vaccine [41].

\section{Chikungunya}

Chikungunya is an alphavirus transmitted by $A$. aegypti mosquitos. From 2013 to 2015, it spread throughout 45 countries in tropical and subtropical North and South America, with over 1.7 million reported cases of infection. Infection is often clinically symptomatic, with febrile illness and debilitating polyarthralgia, hence the derivation of the name "chikungunya" from the phrase "that which bends" in the Makonde language of Tanzania [28]. Though mortality is low compared with other arboviral diseases $(<1 \%)$, morbidity can be significant, in the form of persistent polyarthralgia. For instance, there are reports of up to $60 \%$ of survivors suffering from joint pain three years post-infection [42]. There are also reported neurologic complications, including meningoencephalitis, myelitis, and Guillain-Barré syndrome. In a retrospective study following a 2006 outbreak in western India, Chandak et al. [43] reported that 49 of 300 (16\%) patients had neurological complications, including over half with encephalitis and the remaining with myelopathy, peripheral neuropathy, and myeloneuropathy.

There are no licensed vaccines for chikungunya, though more than 15 candidates are under development, including inactivated, live attenuated, chimeric, subunit protein, viruslike particle, and DNA vaccines. Two inactivated whole virus vaccines have reached Phase 1 trials, and one in a measles vector platform has reached Phase 2 trials [42]. Chikungunya vaccine efforts are not entirely new; in the 1980s, the US military developed a live-attenuated vaccine with strong immunogenicity and mild side effects, namely transient arthralgia in approximately $10 \%$ of the recipients. This vaccine was discontinued owing to low priority at the time, but has since been provided to vaccine developers following the chikungunya resurgence in the mid-2000s [42].

\section{Vaccines Available for Rabies Virus}

Although neither a flavi- nor an alphavirus, no discussion of significant neuroinfectious diseases is complete without the mention of rabies. Despite the availability of pre- and postexposure prophylaxis, rabies remains a significant cause of viral encephalitis, responsible for approximately 60,000 deaths annually, particularly in sub-Saharan Africa and India 
[4]. The rabies virus is found in the saliva of many domestic and wild mammals and transmission to humans occurs directly, usually through the bite of an infected animal. Rabies infection results in an acute encephalomyelitis, which is almost always fatal following symptom onset [44]. A less common presentation is paralytic rabies, which closely simulates Guillain-Barré syndrome or ascending myelitis [45].

The neurologic complications of rabies are entirely preventable with prompt post-exposure prophylaxis vaccination, which can also include the administration of human or equine rabies immunoglobulin (RIG). Widespread dog vaccination programs have also proven effective at disease control, but many parts of the developing world lack the infrastructure to widely implement and track dog vaccines [46]. However, the limited supply and high cost of RIG limits its accessibility in low-income, endemic countries, necessitating the development of alternative options.

Recently, De Benedictis et al. [44] identified two broadly neutralizing human monoclonal antibodies from vaccinated donors, and demonstrated that the antibodies were able to neutralize all 35 rabies viruses and 25 nonrabies lyssaviruses, showing higher potency and breadth than other antibodies under clinical development and the currently available RIG [44]. It is thought that infection with all lyssaviruses culminates in viral encephalitides clinically indistinguishable from rabies; therefore, it is significant to identify broadly neutralizing antibodies capable of binding RABV and non-RABV lyssaviruses [44].

\section{The Significance of Vaccines Against Nonzoonotic Agents}

Nonzoonotic viral diseases, unlike those described above, allow for the tantalizing possibility of global eradication. Without an environmental reservoir, an eradicated pathogen cannot re-emerge unless intentionally reintroduced by humans. Worldwide vaccination programs have enabled the total eradication of the variola major and minor viruses, the causative agents of smallpox. The last documented case of smallpox was in 1977, and hence vaccination efforts have been discontinued. The eradication of poliovirus, which causes irreversible paralysis in up to $1 \%$ cases, is in sight. With the inactivated and live oral poliovirus vaccines, significant progress has been made towards global eradication, with 359 reported cases of wild polio ( $v s$ vaccine-derived) in 2014 [1], 74 cases in 2015 [47], and only nine cases in the first four months of 2016. In the posteradication era, inactivated vaccines based on attenuated strains are preferred from a biosafety standpoint to any live formulations. Sanders et al. [47] recently described several stably attenuated, immunogenic poliovirus strains capable of propagating at $30^{\circ} \mathrm{C}$ but not $37^{\circ} \mathrm{C}$, suggesting that they are noninfectious at physiologic temperatures, thereby decreasing the biosafety risks associated with manufacturing.

\section{Vaccines Available for Measles Virus}

Subacute sclerosing panencephalitis (SSPE) is a fatal progressive neurologic disorder that can manifest $6-8$ years after a clinical measles infection. Symptoms may begin subtly, with behavioral changes and intellectual decline, and progress to generalized convulsions, dementia, and death. Data from the UK and USA place the incidence of SSPE at 4-11 cases per 100,000 cases of measles [48].

The currently approved measles vaccines are live attenuated, either monovalent or multivalent, administered alongside one or more vaccines against mumps, rubella, and varicella [11]. Epidemiologic data following the introduction of the measles, mumps, and rubella vaccine demonstrate a marked decrease of neurologic events associated with these agents [49]. In countries with good measles control through vaccination, a decline in new SSPE cases is observable a few years following a decline in measles incidence $[48,50]$ and when epidemics occur, an increase in SSPE is observable after a few years. Measles vaccinations, combined with surveillance and SSPE registers, have reduced the incidence of SSPE by 82 $96 \%$ in the UK, USA, the Netherlands, Israel, Poland, and Bulgaria [48].

Measles was declared eliminated in the USA in 2000; however, the recent re-emergence is closely tied to parental opposition to vaccination. In a review of 18 measles studies published between 2000 and 2015, Phadke et al. [51] found that of the 1416 measles cases, $56 \%$ had no history of vaccination and $41 \%$ had nonmedical exemptions. Such interference should not be permitted in an age armed with the technological means to eradicate human disease.

\section{Vaccines Under Development for Human Herpesviruses}

The human herpesviruses (HHVs) account for a significant fraction of viral-associated neurologic diseases, both due to primary infection and reactivation from latency. For instance, herpes simplex virus (HSV) is the most common identifiable cause of viral encephalitis, responsible for about $13.8 \%$ of all cases [52]. Approximately $90 \%$ cases of HSV encephalitis are caused by HSV-1, and $10 \%$ by HSV-2. HSV encephalitis is reported to result in neurocognitive sequelae, predominantly memory impairment [4], in up to $70 \%$ survivors. Encephalitis can result from a primary infection, re-infection, or reactivation of latent virus. Reactivation of HSV and other HHVs is often tied to the suppression of cell-mediated immunity, whether by immunosuppression or age-related immunosenescence [4]. HHV-6 is another HHV with increasingly recognized encephalitic potential, particularly in the setting of clinical immunosuppression [53], as well as primary 
infection in young children [54]. A wide variety of neurologic presentations are also associated with Epstein-Barr virus (EBV), including Guillain-Barré syndrome, cranial nerve palsy, optic neuritis, encephalitis, and meningitis. Encephalitis/ meningitis was in fact the first described neurologic complication of infectious mononucleosis (IM), a symptomatic primary infection with EBV [55]. The frequency of neurologic involvement in IM is estimated around 1-18 \%. EBVassociated CNS lymphoma, which is more commonly observed in immunocompromised patients, represents another significant EBV-related CNS disease [56].

Varicella is the only HHV for which there is a currently available vaccine. The formulation is live-attenuated, which can prevent primary infection as well as reactivation in older adults [57]. Not yet available for clinical use is an adjuvanted recombinant subunit formulation [58]. Similar to the other HHVs, primary infection with varicella has been associated with CNS complications such as ataxia, encephalitis, stroke, meningitis, and Guillain-Barré syndrome [59].

Vaccine development for HSV-2 is being actively pursued, as HSV-2 is the leading cause of genital ulcer disease and increases the risk of acquiring HIV. Both preventative and therapeutic strategies are being explored. The most advanced in clinical trials are adjuvanted glycoprotein (gp) subunit vaccines, as viral surface glycoproteins are rationale targets for neutralizing antibodies. Also in preclinical phases are several live attenuated or replication defective candidates [60]. Vaccines for EBV are also under active development, as $\mathrm{EBV}$ is a recognized oncovirus and is annually associated with about 200,000 cases of cancer worldwide [61]. There has been one Phase 2 trial of an EBV vaccine in humans, involving 181 seronegative young adults. Those who received the vaccine of soluble adjuvanted gp350 had a $78 \%$ reduction in the rate of IM, but no difference in the rate of infection. Phase 3 studies are planned to test efficacy in a larger cohort [62]. A similar study was performed in China with seronegative infants, and only one-third of the vaccinated children became infected at 16 months of age, compared with all of the unvaccinated controls. The vaccine in this particular study was a vaccinia virus expressing EBV gp350 [61]. Though for clinical trials the former study in young adults is more feasible, the latter study in infants is the ideal strategy for ubiquitous HHVs acquired at a young age, as the most effective mechanism to prevent HHV-associated diseases would be the complete prevention of infection [62].

\section{Nonviral Neuroinfectious Agents}

While viruses represent a substantial proportion of the inciting agents of neuroinflammation, there are indeed bacterial, fungal, and protozoal agents that contribute to the global burden of neuroinfectious disease. The following section discusses a single agent from each of the above categories, for which vaccines are at various stages of development and implementation.

\section{Bacterial: Neisseria meningitides}

Meningococcal disease is caused by certain serogroups of the Gram-negative proteobacteria Neisseria meningitides [63]. Sporadic cases are found worldwide, with large outbreaks occurring in the "meningitis belt" of sub-Saharan Africa, often during the November to June dry season. Humans are the only natural reservoir, and transmission occurs through direct contact and respiratory droplets [11]. Though $N$. meningitides is a human commensal, in rare cases it can proliferate in the CNS, with infection resulting in meningitis or meningoencephalitis, which is fatal in 5-10\% cases [11]. Permanent neurologic sequelae are common among survivors [63].

The currently available $N$. meningitides vaccines include polysaccharide and conjugate formulations. Polysaccharide vaccines are lyophilized, heat-stable purified capsular polysaccharides from meningococci of defined serogroups (A, C, $\mathrm{Y}$, and W-135), available as bi-, tri-, or tetravalent vaccines. However, these have been largely replaced by multivalent conjugate vaccines, in which polysaccharides from the above serogroups are conjugated to a carrier protein to induce a $\mathrm{T}$ cell-dependent immune response [19]. The conjugate vaccines are serogroup-specific and highly immunogenic, capable of eliciting humoral responses even in infants. The vaccine is recommended for travelers from low-endemic regions who are planning to visit highly endemic regions [11]. Guidelines were recently issued for another vaccine that was licensed by the US Food and Drug Administration in 2014, and provides protection against the fifth serogroup of $N$. meningitides, serogroup B [64].

\section{Fungal: Cryptococcus}

Fungi can trigger meningitis in immunocompromised patients, though this is far less common in immunocompetent individuals. Cryptococcus is the most common cause of fungal meningitis and meningoencephalitis. Global estimates suggest that 1 million cases of cryptococcal meningitis occur each year, with approximately 600,000 fatalities [65]. CNS disease resulting from $C$. neoformans strains are most common in patients with advanced HIV, while $C$. gattii strains are emerging pathogens in immunocompetent individuals [66, 67].

Humans typically encounter fungi via spore inhalation or a breach in anatomical barriers, as occurs in nosocomial fungal infections [67]. Opportunistic fungal infections often occur in immunosuppressed patients, such as those undergoing treatments for cancer, transplant, autoimmune disease, or HIV. Owing to the HIV pandemic, fungal infections in CD4 
lymphopenic patients have risen rapidly, and carry a mortality rate of $50-70 \%$ [67]. Consequently, there is an emphasis on understanding the role played by CD8 cells in antifungal immunity, and the design of antifungal vaccines to augment memory CD8 responses [67].

At present, there are no available antifungal vaccines, despite promising preclinical reports. A 2011 study demonstrated that when immunocompetent mice were immunized with an interferon-gamma-producing strain of $C$. neoformans (H99 $\gamma$ ) and subsequently rendered CD4 and CD8 T cell deficient, the vaccine conferred protection for up to 100 days postimmunization [68]. Separately, a recent report by Rella et al. [69] describes a nonpathogenic sterylglucoside mutant strain of $C$. neoformans that conferred complete protection against lethal doses of both $C$. neoformans and $C$. gattii in mice. The authors went on to show that pretreatment with this mutant strain in CD4 $\mathrm{T}$ cell-depleted mice similarly protected against subsequent lethal challenge with $C$. neoformans [69].

Though these and several other studies somewhat account for a context of CD4 deficiency, many employ live-attenuated fungal strains, which pose a risk in immunocompromised individuals. However, from these early foundational studies, alternate formulations can be developed, such as heat-killed strains or vesicles, which would eliminate the hazards associated with a live vaccine [69]. Another research priority for the field is the identification of protective CD8 T cell antigens [67].

\section{Protozoal: Plasmodium}

Malaria remains one of the deadliest infectious diseases in tropical countries, with 214 million cases and 438,000 deaths in 2015 alone [70]. More than $90 \%$ of the deaths occur in sub-Saharan Africa, and many in children younger than 5 years [71], though this is shifting to school-age children [72]. It involves infection of human erythrocytes by the protozoan Plasmodium, transmitted by Anopheles mosquitos. There are five Plasmodium species known to infect humans and the most severe cases, including cerebral malaria $(\mathrm{CM})$, are caused by Plasmodium falciparum [73]. CM is defined as a diffuse encephalopathy, with seizures and coma as the clinical hallmark. The associated mortality rate is around $8 \%$ but can reach $50 \%$ in patients with multiple organ dysfunction [73], which is also associated with P. falciparum infection [71]. Short- and longterm neurologic complications are common among children with CM. Deficits such as ataxia, paresis, epilepsy, disruptive behavior, and cognitive impairment have been reported to resolve within 6 months of infection; however, another study reported persistent neurologic and cognitive deficits for up to 9 years following infection. Intracranial hypertension is thought to be associated with the morbidity and mortality of CM.
There is currently no available malaria vaccine, though over 30 P. falciparum candidates are at advanced preclinical or clinical stages [71]. Vaccine development efforts have mainly focused on P. falciparum, followed by P. vivax, which is the dominant species outside sub-Saharan Africa [71].

A large Phase 3 trial was recently completed with candidate RTS, S/AS01 (Mosquirix), a recombinant fusion protein expressing regions of the $P$. falciparum circumsporozoite protein covalently bound to the hepatitis B surface antigen. This fusion protein is expressed with hepatitis B surface antigen to form virus-like particles, which are reconstituted in an adjuvant to form the vaccine suspension. The Phase 3 trial evaluated two groups of children. Eighteen months after the third dose, the efficacy was estimated at $45 \%$ for the 5-17 montholds and $26 \%$ for the $6-12$ week-olds. When the whole trial period was evaluated, the efficacy was $26 \%$ and $18 \%$, respectively [71]. This limited efficacy underscores the challenge of developing a vaccine against a complex organism with an array of stage-specific genes [74], and while more safety and efficacy data are clearly needed, RTS, S/AS01 represents the first malaria vaccine with a favorable benefit-risk profile [75]. However, there are strategies to take advantage of the short-term protection of this vaccine, such as reactive ring vaccinations, whereby all people within a radius around a geographically defined population are vaccinated. Similar approaches proved key to the eradication of smallpox [72].

\section{Looking Forward}

Research efforts to stem the tide of infection-associated neurologic complications will at times be reactionary, particularly to newly emerging neuroinfectious disease, such as the flaccid paralysis associated with the 2014 outbreak of enterovirus D68 and the even more recently emerged Zika pandemic. In an editorial on Zika virus, Dr. Anthony Fauci writes that we should prepare for a continual emergence of arthropod-borne diseases stemming from behaviors that perturb the ecologic balance, including global warming, increasing urbanization, and international travel [6]. However, with concrete steps, the time to develop and deploy effective vaccine therapies can be substantially reduced. Such steps include the development of cross-protective therapies and easily modifiable immunogenic vaccine platforms, the increase of international stockpiles, and the acceleration of vaccination campaigns in endemic areas [17].

Many of the current efficacious vaccines are liveattenuated formulations, which mimic natural infection through a biologically relevant (albeit controlled) amplification of the agent [24]. As live-attenuated vaccines elicit some safety concerns, particularly in subpopulations such as pregnant women and immunocompromised individuals, subunit 
vaccines with various prime/boost strategies may offer a solution, though vaccine strategies must be agent-dependent [76]. Given the worldwide morbidity and mortality directly stemming from CNS infections, vaccine strategies against neuroinfectious agents must be made a global health priority.

Required Author Forms Disclosure forms provided by the authors are available with the online version of this article.

\section{References}

1. Andre, F.E. et al. Vaccination greatly reduces disease, disability, death and inequity worldwide. Bull World Health Organ 86, 140-146 (2008).

2. Nath, A. \& Tyler, K.L. Novel approaches and challenges to treatment of central nervous system viral infections. Ann Neurol 74, 412-422 (2013).

3. Tracey, J. Syndromic Approach to Neuroinfectious disease (ed. Kelly D Flemming, L.K.J.) (Oxford University Press, 2015).

4. John, C.C. et al. Global research priorities for infections that affect the nervous system. Nature 527, S178-S186 (2015).

5. Go, Y.Y., Balasuriya, U.B. \& Lee, C.K. Zoonotic encephalitides caused by arboviruses: transmission and epidemiology of alphaviruses and flaviviruses. Clin Exp Vaccine Res 3, 58-77 (2014).

6. Fauci, A.S. \& Morens, D.M. Zika Virus in the Americas-yet another arbovirus threat. $N$ Engl J Med 374, 601-604 (2016).

7. Liang, H., Lee, M. \& Jin, X. Guiding dengue vaccine development using knowledge gained from the success of the yellow fever vaccine. Cell Mol Immunol 13, 36-46 (2016).

8. Wahala, W.M., Kraus, A.A., Haymore, L.B., Accavitti-Loper, M.A. $\&$ de Silva, A.M. Dengue virus neutralization by human immune sera: role of envelope protein domain III-reactive antibody. Virology 392, 103-113 (2009).

9. Erra, E.O. \& Kantele, A. The Vero cell-derived, inactivated, SA1414-2 strain-based vaccine (Ixiaro) for prevention of Japanese encephalitis. Expert Rev Vaccines 14, 1167-1179 (2015).

10. Hills, S.L., Rabe, I.B., Fischer, M. Japanese Encephalitis, Chapter 3: Infectious Diseases Related to Travel. http://www.cdc.gov (2015 ) .

11. Vaccine-preventable diseases and vaccines. WHO International Travel and Health publication (2015).

12. Heinz, F.X. et al. Vaccination and tick-borne encephalitis, central Europe. Emerg Infect Dis 19, 69-76 (2013).

13. Zajkowska, J. et al. Fatal outcome of tick-borne encephalitis-a case series. Neurol Neurochir Pol 45, 402-6 (2011).

14. Veje, M. et al. Tick-Borne encephalitis sequelae at long-term followup: a self-reported case-control study. Acta Neurol Scand (2016).

15. Kunze, U. \& Kunze, M. The Austrian vaccination paradox: tickborne encephalitis vaccination versus influenza vaccination. Cent Eur J Public Health 23, 223-226 (2015).

16. Karunamoorthi, K. Yellow fever encephalitis: an emerging and resurging global public health threat in a changing environment (ed. S., T.) (2013).

17. Butler, D. Fears rise over yellow fever's next move. Nature $\mathbf{5 3 2}$ 155-156 (2016).

18. Meeting of the Strategic Advisory Group of Experts on immunization, April 2013-conclusions and recommendations. Wkly Epidemiol Rec 88, 201-206 (2013).

19. Li, S. et al. Molecular signatures of antibody responses derived from a systems biology study of five human vaccines. Nat Immunol 15, 195-204 (2014).

20. Petrovsky, N. et al. An inactivated cell culture Japanese encephalitis vaccine (JE-ADVAX) formulated with delta inulin adjuvant provides robust heterologous protection against West Nile encephalitis via cross-protective memory B cells and neutralizing antibody. J Virol 87, 10324-10333 (2013).

21. Lobigs, M. \& Diamond, M.S. Feasibility of cross-protective vaccination against flaviviruses of the Japanese encephalitis serocomplex. Expert Rev Vaccines 11, 177-187 (2012).

22. Barrett, P.N., Mundt, W., Kistner, O. \& Howard, M.K. Vero cell platform in vaccine production: moving towards cell culture-based viral vaccines. Expert Rev Vaccines 8, 607-618 (2009).

23. Hotez, P.J. Zika in the United States of America and a Fateful 1969 Decision. PLoS Negl Trop Dis 10, e0004765 (2016).

24. Whitehead, S.S. Development of TV003/TV005, a single dose, highly immunogenic live attenuated dengue vaccine; what makes this vaccine different from the Sanofi-Pasteur CYD vaccine? Expert Rev Vaccines 15, 509-517 (2016).

25. Wilder-Smith, A. \& Yoon, I.K. Edging closer towards the goal of a dengue vaccine. Expert Rev Vaccines 15, 433-435 (2016).

26. Broutet, N. et al. Zika Virus as a Cause of Neurologic Disorders. $N$ Engl J Med (2016).

27. Tripp, R.A. \& Ross, T.M. Development of a Zika vaccine. Expert Rev Vaccines, 1-3 (2016).

28. Pastula, D.M., Smith, D.E., Beckham, J.D. \& Tyler, K.L. Four emerging arboviral diseases in North America: Jamestown Canyon, Powassan, chikungunya, and Zika virus diseases. $J$ Neurovirol (2016).

29. Petersen, L.R., Jamieson, D.J., Powers, A.M. \& Honein, M.A. Zika virus. $N$ Engl J Med (2016).

30. Schuler-Faccini, L. et al. Possible association between Zika virus infection and microcephaly-Brazil, 2015. MMWR Morb Mortal Wkly Rep 65, 59-62 (2016).

31. Malkki, H. CNS infections: Zika virus infection could trigger Guillain-Barré syndrome. Nat Rev Neurol 12, 187 (2016).

32. Cao-Lormeau, V.M. et al. Zika virus, French polynesia, South pacific, 2013. Emerg Infect Dis 20, 1085-1086 (2014).

33. Carteaux, G. et al. Zika virus associated with meningoencephalitis. N Engl J Med (2016).

34. Mecharles, S. et al. Acute myelitis due to Zika virus infection. Lancet (2016).

35. Bell, T.M., Field, E.J. \& Narang, H.K. Zika virus infection of the central nervous system of mice. Arch Gesamte Virusforsch 35, 183 193 (1971).

36. Palacios, R., Poland, G.A. \& Kalil, J. Another emerging arbovirus, another emerging vaccine: targeting Zika virus. Vaccine (2016).

37. Zacks, M.A. \& Paessler, S. Encephalitic alphaviruses. Vet Microbiol 140, 281-286 (2010).

38. Guerbois, M. et al. IRES-driven expression of the capsid protein of the Venezuelan equine encephalitis virus TC-83 vaccine strain increases its attenuation and safety. PLoS Negl Trop Dis 7, e2197 (2013).

39. Molina, O.M. et al. [Venezuelan equine encephalitis. 1995 outbreak: clinical profile of the case with neurologic involvement]. Rev Neurol 29, 296-298 (1999).

40. Paessler, S. et al. Recombinant sindbis/Venezuelan equine encephalitis virus is highly attenuated and immunogenic. $J$ Virol 77, 92789286 (2003).

41. Phillips, A.T. et al. Liposome-antigen-nucleic acid complexes protect mice from lethal challenge with western and eastern equine encephalitis viruses. $J$ Virol 88, 1771-1780 (2014).

42. Smalley, C., Erasmus, J.H., Chesson, C.B. \& Beasley, D.W. Status of research and development of vaccines for chikungunya. Vaccine (2016).

43. Chandak, N.H. et al. Neurological complications of Chikungunya virus infection. Neurol India 57, 177-180 (2009).

44. De Benedictis, P. et al. Development of broad-spectrum human monoclonal antibodies for rabies post-exposure prophylaxis. EMBO Mol Med (2016). 
45. Kalita, J. et al. Paralytic rabies: MRI findings and review of literature. Neurol India 62, 662-664 (2014).

46. Hampson, $\mathrm{K}$. et al. Estimating the global burden of endemic canine rabies. PLoS Negl Trop Dis 9, e0003709 (2015).

47. Sanders, B.P. et al. Cold-Adapted viral attenuation (CAVA): highly temperature sensitive polioviruses as novel vaccine strains for a next generation inactivated poliovirus vaccine. PLoS Pathog 12, e1005483 (2016).

48. Campbell, H., Andrews, N., Brown, K.E. \& Miller, E. Review of the effect of measles vaccination on the epidemiology of SSPE. Int $J$ Epidemiol 36, 1334-1348 (2007).

49. Gualberto, F.A. et al. Fulminant encephalitis associated with a vaccine strain of rubella virus. J Clin Virol 58, 737-740 (2013).

50. Subacute sclerosing panencephalitis and measles vaccination. Wkly Epidemiol Rec (2006).

51. Phadke, V.K., Bednarczyk, R.A., Salmon, D.A. \& Omer, S.B. Association between vaccine refusal and vaccine-preventable diseases in the United States: a review of measles and pertussis. JAMA 315, 1149-1158 (2016).

52. George, B.P., Schneider, E.B. \& Venkatesan, A. Encephalitis hospitalization rates and inpatient mortality in the United States, 2000 2010. PLoS One 9, e104169 (2014).

53. Ogata, M., Fukuda, T. \& Teshima, T. Human herpesvirus-6 encephalitis after allogeneic hematopoietic cell transplantation: what we do and do not know. Bone Marrow Transplant 50, 1030-1036 (2015).

54. Tesini, B.L., Epstein, L.G. \& Caserta, M.T. Clinical impact of primary infection with roseoloviruses. Curr Opin Virol 9, 91-96 (2014).

55. Connelly, K.P. \& DeWitt, L.D. Neurologic complications of infectious mononucleosis. Pediatr Neurol 10, 181-184 (1994).

56. Weinberg, A., Li, S., Palmer, M. \& Tyler, K.L. Quantitative CSF PCR in Epstein-Barr virus infections of the central nervous system. Ann Neurol 52, 543-548 (2002).

57. Johnston, C., Koelle, D.M. \& Wald, A. HSV-2: in pursuit of a vaccine. J Clin Invest 121, 4600-4609 (2011).

58. Gagliardi, A.M., Andriolo, B.N., Torloni, M.R. \& Soares, B.G. Vaccines for preventing herpes zoster in older adults. Cochrane Database Syst Rev 3, CD008858 (2016)

59. Science, M. et al. Central nervous system complications of varicella-zoster virus. J Pediatr 165, 779-785 (2014).

60. Johnston, C., Gottlieb, S.L. \& Wald, A. Status of vaccine research and development of vaccines for herpes simplex virus prepared for WHO PD-VAC. Vaccine (2016).
61. Cohen, J.I., Mocarski, E.S., Raab-Traub, N., Corey, L. \& Nabel, G.J. The need and challenges for development of an Epstein-Barr virus vaccine. Vaccine 31(Suppl. 2), B194-B196 (2013).

62. Smith, C. \& Khanna, R. The development of prophylactic and therapeutic EBV vaccines. Curr Top Microbiol Immunol 391, 455-473 (2015).

63. Gianchecchi, E., Torelli, A., Piccini, G., Piccirella, S. \& Montomoli, E. Neisseria meningitidis infection: who, when and where? Expert Rev Anti Infect Ther 13, 1249-1263 (2015).

64. Folaranmi, T. et al. Use of serogroup B meningococcal vaccines in persons aged $>/=10$ years at increased risk for serogroup B meningococcal disease: recommendations of the Advisory Committee on Immunization Practices, 2015. MMWR Morb Mortal Wkly Rep 64, 608-612 (2015).

65. Park, B.J. et al. Estimation of the current global burden of cryptococcal meningitis among persons living with HIV/AIDS. AIDS 23, 525-530 (2009).

66. Wilson, J.W. Fungal infections of the central nervous system (Mayo Foundation for Medical Education and Research, 2015).

67. Nanjappa, S.G. \& Klein, B.S. Vaccine immunity against fungal infections. Curr Opin Immunol 28, 27-33 (2014).

68. Wozniak, K.L., Young, M.L. \& Wormley, F.L., Jr. Protective immunity against experimental pulmonary cryptococcosis in T celldepleted mice. Clin Vaccine Immunol 18, 717-723 (2011).

69. Rella, A. et al. Role of Sterylglucosidase 1 (Sgl1) on the pathogenicity of Cryptococcus neoformans: potential applications for vaccine development. Front Microbiol 6, 836 (2015).

70. Dieye, Y. et al. Cytokine response during non-cerebral and cerebral malaria: evidence of a failure to control inflammation as a cause of death in African adults. PeerJ 4, e1965 (2016).

71. Malaria vaccine: WHO position paper-January 2016. Wkly Epidemiol Rec 91, 33-51 (2016).

72. Gosling, R. \& von Seidlein, L. The future of the RTS,S/AS01 malaria vaccine: an alternative development plan. PLoS Med 13, e1001994 (2016)

73. Hora, R., Kapoor, P., Thind, K.K. \& Mishra, P.C. Cerebral malaria clinical manifestations and pathogenesis. Metab Brain Dis 31, 225 237 (2016).

74. Long, C.A. \& Zavala, F. Malaria vaccines and human immune responses. Curr Opin Microbiol 32, 96-102 (2016).

75. Morrison, C. Landmark green light for Mosquirix malaria vaccine. Nat Biotechnol 33, 1015-1016 (2015).

76. Kardani, K., Bolhassani, A. \& Shahbazi, S. Prime-boost vaccine strategy against viral infections: mechanisms and benefits. Vaccine 34, 413-423 (2016) 